\section{Korean Guidelines for Cardiopulmonary Resuscitation. Part 3. Adult basic life support}

Kyoung-Jun Song', Sun Young Lee ${ }^{2,3}$, Gyu Chong $\mathrm{Cho}^{4}$, Giwoon $\mathrm{Kim}^{5}$, Jung-Youn $\mathrm{Kim}^{6}$, Jaehoon $\mathrm{Oh}^{7}$, Je Hyeok $\mathrm{Oh}^{8}$, Seung $\mathrm{Ryu}^{9}$, Seung Mok Ryoo ${ }^{10}$, Eun-Ho Lee ${ }^{11}$, Sung Oh Hwang ${ }^{12}$, Ju Young Hong ${ }^{13}$, Sung Phil Chung ${ }^{13}$

\footnotetext{
'Department of Emergency Medicine, SMG-SNU Boramae Medical Center, Seoul, Korea

${ }^{2}$ Public Healthcare Center, Seoul National University Hospital, Seoul, Korea

${ }^{3}$ Laboratory of Emergency Medical Services, Seoul National University Hospital Biomedical Research Institute, Seoul, Korea

${ }^{4}$ Department of Emergency Medicine, Hallym University College of Medicine, Chuncheon, Korea

${ }^{5}$ Department of Emergency Medicine, Soonchunhyang University Bucheon Hospital, Bucheon, Korea

${ }^{6}$ Department of Emergency Medicine, Korea University College of Medicine, Seoul, Korea

${ }^{7}$ Department of Emergency Medicine, Hanyang University College of Medicine, Seoul, Korea

${ }^{8}$ Department of Emergency Medicine, Chung-Ang University College of Medicine, Seoul, Korea

${ }^{9}$ Department of Emergency Medicine, Chungnam National University Hospital, Daejeon, Korea

${ }^{10}$ Department of Emergency Medicine, Asan Medical Center, University of Ulsan College of Medicine, Seoul, Korea

${ }^{11}$ Department of Anesthesiology and Pain Medicine, Asan Medical Center, University of Ulsan College of Medicine, Seoul, Korea

${ }^{12}$ Department of Emergency Medicine, Yonsei University Wonju College of Medicine, Wonju, Korea

${ }^{13}$ Department of Emergency Medicine, Yonsei University College of Medicine, Seoul, Korea
}

\section{MAJOR UPDATE OF THE 2020 KOREAN BASIC LIFE SUPPORT GUIDELINES}

This article presents the update of the adult basic life support (BLS) of the 2015 Korean Guidelines for Cardiopulmonary Resuscitation. 'The class of recommendations and level of evidence are written according to the "2020 Korean Guidelines for Cardiopulmonary Resuscitation. Part 1. The update process and highlights". The main new or revised contents in the 2020 Korean BLS guidelines are as follows.

Reinforced role of the emergency medical dispatcher

Implementing a dispatcher-assisted cardiopulmonary resuscitation (CPR) program can be effective in improving the survival outcomes of adult victims of cardiac arrest. It is recommended that emergency medical dispatchers (EMDs) apply standardized algorithms to quickly detect cardiac arrest when receiving an emergency call.

Change of the 2015 BLS guidelines

If the rescuer is alone and has a mobile phone, he/she should start CPR immediately after turning on the speaker of the mobile phone or activating the hands-free function and getting assis-
elSSN: 2383-4625

Received: 7 March 2021

Revised: 7 March 2021

Accepted: 19 March 2021

Correspondence to: Sung Phil Chung Department of Emergency Medicine, Yonsei University College of Medicine, 211 Enoju-ro, Gangnam-gu,

Seoul 06273, Korea

E-mail:emstar@yuhs.ac

ORCID

https://orcid.org/0000-0002-3074-011X

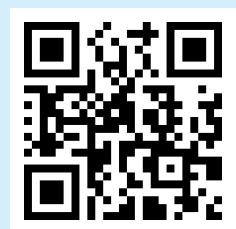

How to cite this article:

Song KJ, Lee SY, Cho GC, Kim G, Kim JY, Oh J, Oh JH, Ryu S, Ryoo SM, Lee EH, Hwang SO, Hong JY, Chung SP. 2020 Korean Guidelines for Cardiopulmonary Resuscitation. Part 3. Adult basic life support. Clin Exp Emerg Med 2021;8(S):S15-S25. https://doi.

org/10.15441/ceem.21.024

This is an Open Access article distributed under the terms of the Creative Commons Attribution Non-Commercial License (https:// creativecommons.org/licenses/by-nc/4.0/). 
tance from an EMD. If the victim is not lying on a bed, he/she should be placed on a hard floor, and chest compressions should be performed; if he/she is lying on a bed in a hospital, a backboard should be inserted between the mattress and his/her back and chest compressions should be performed. The victim should not be moved from a bed to the floor to improve the depth of chest compressions. For adult victims, chest compressions should be restarted immediately after defibrillation. The rescuer could consider stopping chest compressions every two minutes to check the heart rhythm. Chest compression can be temporarily stopped to check the rhythm when physiological indicators that suggest return of spontaneous circulation (ROSC) (waveforms in the artery or rapid increase in end-expiratory carbon dioxide) are noted.

\section{Duration of cardiopulmonary resuscitation on the scene} Emergency medical technicians (EMTs) should consider hospital transfer if ROSC does not occur after on-site BLS is performed for 6 minutes. EMTs should consider hospital transfer if ROSC does not occur after on-site advanced resuscitation is performed for 10 minutes; however, they may consider extending the duration based on the medical directions of a physician.

\section{Airway obstruction by a foreign body}

Tapping on the back is recommended as a priority treatment for patients with airway obstruction caused by a foreign body and for patients who are unable to cough effectively. The abdominal push method is recommended when back tapping is not effective.

\section{Drowning patient}

Bystander CPR should be performed for victims of cardiac arrest as a result of drowning. If the rescuer is trained and willing to perform, standard CPR including ventilation should be performed. The use of an automated external defibrillator (AED) can be considered for victims of cardiac arrest as a result of drowning. A well-trained aquatic rescue team with appropriate equipment may consider underwater CPR and CPR on a lifeboat. It is suggested that immersion time and not age, emergency medical system (EMS) response time, water type (fresh or salt water), water temperature, or witnessed status of the victim, could be used as a prognostic indicator when making search and rescue decisions for drowning victims.

\section{Cardiopulmonary resuscitation for persons suspected to have coronavirus disease \\ Regarding the current coronavirus disease 2019 (COVID-19) pan- demic, chest compressions and CPR can trigger the formation of aerosols, increasing the risk of transmission of infection. Health-}

care providers should wear appropriate personal protective equipment, including masks, gloves, and goggles. If defibrillation is necessary, it should be actively performed while paying attention to the transmission of infection. Chest compression should be performed with the victim's mouth and nose covered as much as possible because even though the risk of infection transmission is low, there is still potential for aerosol generation.

\section{ADULT BLS ALGORITHMS}

In the 2020 guidelines, the adult BLS algorithms are classified according to location and rescuer: out-of-hospital algorithm for lay rescuers, out-of-hospital algorithm for healthcare providers, and in-hospital algorithm for healthcare providers.

\section{Adult out-of-hospital cardiac arrest (OHCA) algorithm for lay rescuers}

The first step of BLS is to check for responsiveness. The safety of the scene needs to be assessed first before approaching the collapsed person. If the collapsed person is unresponsive, a 119 emergency call and a request for an AED should be made immediately, and the rescuer should follow the instructions of the EMD. If normal breathing is observed, the rescuer should continue observing the patient and wait for the EMTs to arrive. If a patient has apnea or abnormal respiration (e.g., agonal respiration), the rescuer should start compression-only CPR. Standard CPR (chest compression: ventilation $=30: 2$ ) is recommended if the rescuer knows how to ventilate and is willing to provide ventilation. If the rescuer can use an AED, defibrillation should be performed according to the voice instructions of the AED. If defibrillation is not required or immediately after defibrillation, CPR should be performed for 2 minutes, and cardiac rhythm should be analyzed again with the AED. CPR should be performed until EMTs arrive, the victim moves, or breathing returns to normal (Fig. 1).

\section{Adult OHCA algorithm for healthcare providers}

After making a 119 emergency call for the unresponsive patient, the victim's pulse and breathing should be checked simultaneously within 10 seconds. If there is no pulse or if breathing is not normal, chest compressions should be started with a compression-ventilation ratio of 30:2. When the AED arrives, cardiac rhythm should be analyzed every 2 minutes, and defibrillation should be performed if necessary. If direct medical control is possible, follow the directions, and consider transfer to a hospital after 6 minutes of on-scene CPR (Fig. 2). 


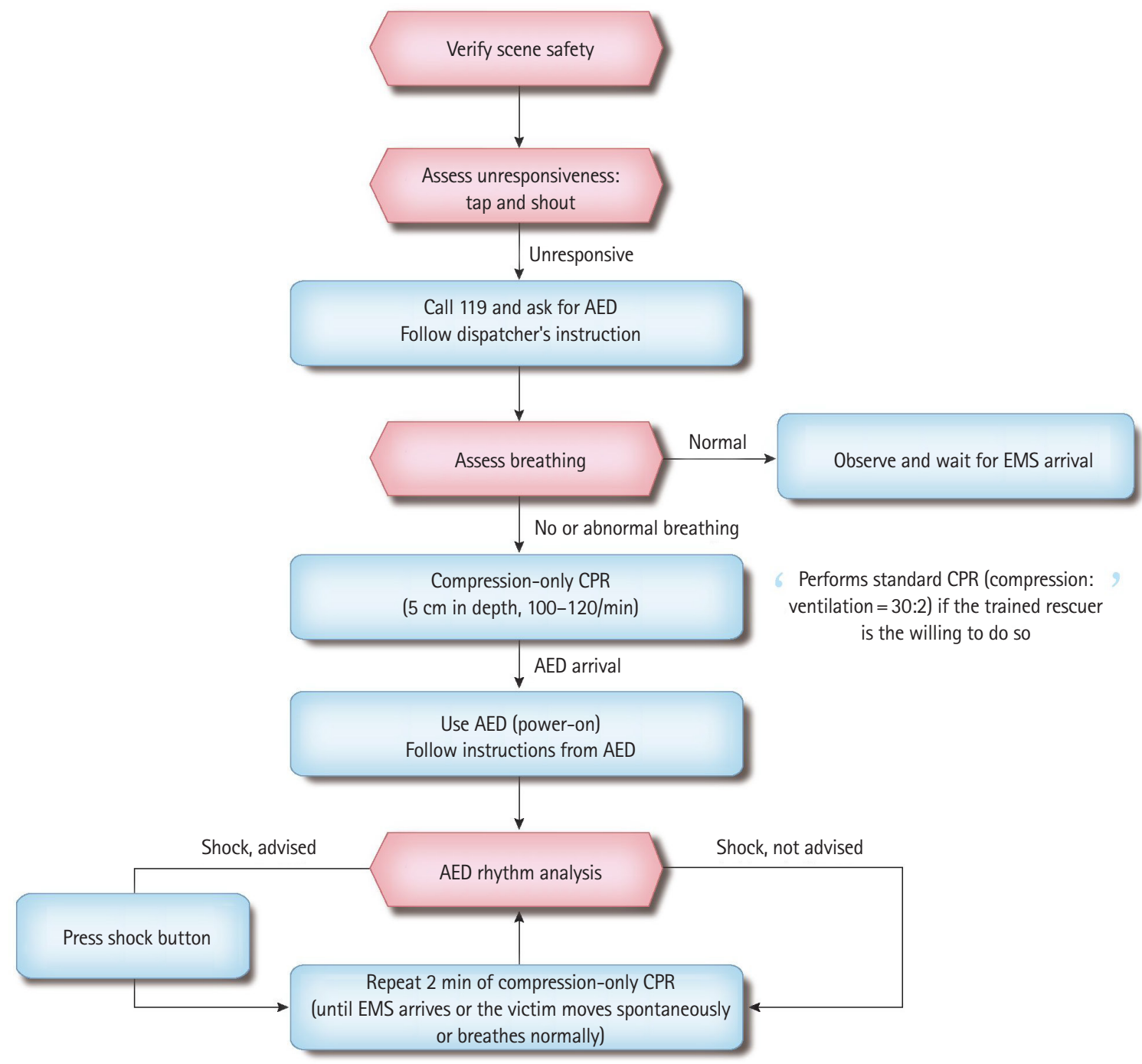

Fig. 1. 2020 Basic life support algorithm for adult out-of-hospital cardiac arrest for lay rescuers. AED, automated external defibrillator; EMS, emergency medical system; CPR, cardiopulmonary resuscitation.

\section{Adult in-hospital cardiac arrest algorithm for healthcare providers}

It is important to prevent the occurrence of cardiac arrest by recognizing early warning signs of cardiac arrest, such as abnormal vital signs. If cardiac arrest is suspected, the healthcare provider should check the victim's responsiveness. If the victim is unresponsive, the healthcare provider should activate the rescue team (rapid response team) in the hospital. Thereafter, the subsequent procedures to be performed are the same as those of the out-ofhospital algorithm for healthcare providers. CPR should be performed until the rapid response team arrives (Fig. 3).

\section{CHECK RESPONSIVENESS}

The safety of the scene and the possibility of infection needs to be assessed first before approaching the collapsed person. Responsiveness can be evaluated by shouting, "Are you alright?" to the collapsed person, and by tapping his/her shoulder.

\section{ACTIVATION OF EMS}

If the collapsed person is unresponsive, a 119 emergency call should be made immediately, and a request for an AED needs to be made. If one witnesses a person collapse, he/she needs to request that someone call 119 by loudly asking others nearby for help; the rescuer should call 119 if there is no one else around. It is challenging for a lay rescuer to precisely evaluate a victim's respiration status. ${ }^{2}$ Thus, a lay rescuer needs the help and guidance of an EMD to determine the respiration status of a patient and to perform compression-only $\mathrm{CPR}^{3}$. Since seizures may be observed in some 


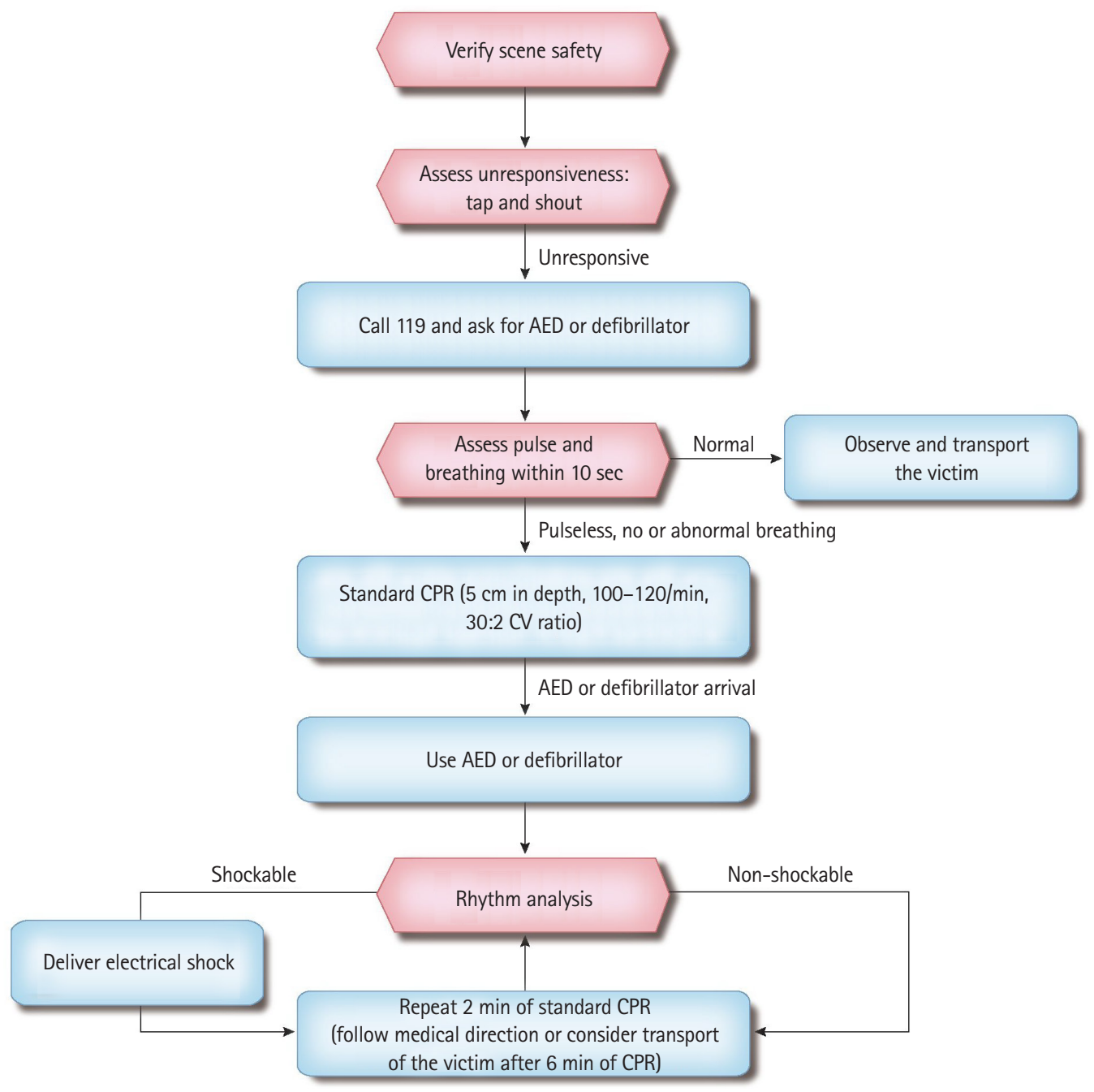

Fig. 2. 2020 Basic life support algorithm for adult out-of-hospital cardiac arrest for healthcare providers. AED, automated external defibrillator; CPR, cardiopulmonary resuscitation; CV, compression-ventilation.

cases, the EMD should be able to reliably identify abnormal breathing and myoclonic muscle spasm and be able to realistically explain these possible scenarios to the rescuer. ${ }^{4}$

\section{Call first regardless of age}

It is difficult to classify and educate the public on different protocols and to know the cause of collapse at the scene. Further, since it is important to shorten the time to arrival at the hospital, rescuers are encouraged to call first and then initiate CPR regardless of the age of the patient.

\section{Call 119}

When calling 119, the location of the incident, the situation, the victim's status, and the emergency treatment being provided need to be reported.' If the rescuer is not trained on CPR or has forgotten the procedure for $\mathrm{CPR}$, he/she should follow the instructions of the EMD. If the rescuer is alone and has a mobile phone, he/ she should start CPR immediately after turning on the speaker of the mobile phone or activating the hands-free function and getting assistance from an EMD. Putting the call on speaker is effective for conducting CPR according to the EMD's instructions.

\section{Dispatcher-assisted CPR}

The EMD can assist a lay rescuer in recognizing cardiac arrest and performing CPR. An EMD should ask the caller if the collapsed person is responsive and if his/her respiration is abnormal. ${ }^{5}$ The EMD determines whether cardiac arrest has occurred if the collapsed person is unresponsive and has apnea or abnormal respiration. If an EMD recognizes cardiac arrest, the dispatcher will provide instructions on how to perform compression-only CPR via telephone. ${ }^{6}$ EMDs providing instructions for CPR to lay rescuers increases bystander CPR rate and improves the survival rate of 


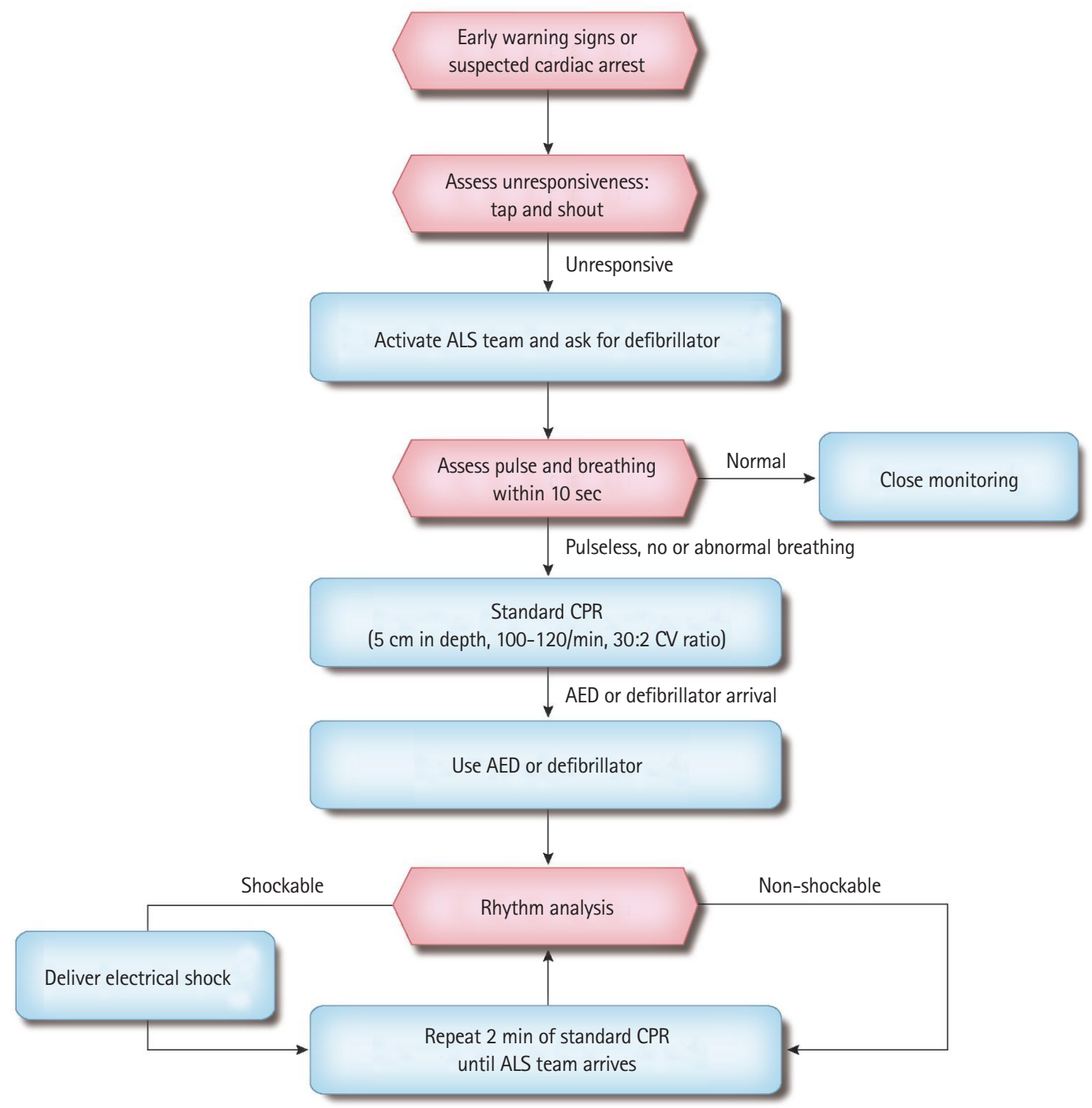

Fig. 3. 2020 Basic life support algorithm for adult in-hospital cardiac arrest. ALS, advanced life support; CPR, cardiopulmonary resuscitation; CV, compression-ventilation; AED, automated external defibrillator.

victims of cardiac arrest. ${ }^{5,7,8}$ It is recommended that the training of EMDs should include how to detect abnormal breathing and how to teach chest compression; they should also be taught that agonal breathing means cardiac arrest and that a convulsive attack may be the first symptom of cardiac arrest. ${ }^{3,4}$ The person in charge of the EMS should shorten the time from the emergency call to the initiation of CPR, evaluate the appropriateness of dispatcher-assisted CPR, and assess the survival outcomes of patients to improve the survival of cardiac arrest. ${ }^{9,10}$ To ensure the successful functioning of the dispatcher-assisted CPR process, a quality control process, including a review of the audio recordings of EMS calls, should be conducted as part of the prehospital EMS quality improvement process. ${ }^{3,11}$

\section{ASSESSMENT OF BREATHING (AND PULSE CHECK)}

The patient's respiration should be evaluated after responsiveness is determined and the 119 call is made. The lay rescuer should initiate chest compressions according to the directions of the EMD if there is abnormal or no breathing. A healthcare provider should check the absence or presence of a pulse and normal respiration within 10 seconds after activating the EMS and asking for an AED. Recognizing agonal breathing as a sign of cardiac arrest is important for rapid CPR and increases the success rate of resuscitation. ${ }^{12-16}$ In the case of normal breathing, the victim should be placed in a recovery position to prevent aspiration of a foreign body in the mouth. To determine the pulse of an adult victim of 
cardiac arrest, the healthcare provider should check the carotid artery or carotid artery plus femoral artery within 10 seconds. $^{17}$

\section{CHEST COMPRESSION}

It is recommended that lay rescuers perform compression-only CPR for adult victims of cardiac arrest. For adults, the depth of compression should be approximately $5 \mathrm{~cm}$, and the rate should be maintained at $100-120 / \mathrm{min}^{18,19}$ The recommended position of one's hand when performing compression is at the lower half of the sternum. The recoil of the chest needs to be maximized after each compression. ${ }^{20,21}$ The suggested compression-ventilation ratio is $30: 2 .{ }^{22-24}$ Considering the high quality of CPR and the fatigue of a rescuer, the role of a compressor needs to be rotated every 2 minutes. ${ }^{17}$

In the 2015 guidelines, it was recommended that lay rescuers should perform chest compression-only CPR, and rescuers who can perform ventilation should perform standard CPR including ventilation. Healthcare providers, including EMTs, should always perform CPR with both compression and ventilation. ${ }^{17,19,25}$ In the case of asphyxial arrest due to drowning or poisoning, or if the rescue happened a long time after cardiac arrest, ventilation should be performed with chest compression.

If an advanced airway is in place, it is suggested that one rescuer continue compression at a rate of 100-120/min without interruption, while the other rescuer provides respiration with a bag valve mask every 6 seconds ( 10 breaths/min).

For adult patients in a hospital, if an advanced airway is in place, it is suggested that the rescuer perform positive pressure ventilation without stopping chest compression. During CPR, the rescuer can stop chest compression every two minutes to check cardiac rhythm. ${ }^{26}$

\section{AIRWAY AND VENTILATION}

For lay rescuers, a trained rescuer can use the head tilt-chin lift maneuver to open the airway. For healthcare providers, if there is no evidence of injury to the head or neck, the airway should always be open, and the position should be maintained by using the head tilt-chin lift maneuver. If a cervical injury is suspected, the jaw thrust technique, which does not include head extension, should be used to open the airway.

We recommend that the patient is ventilated with an inspiration duration of 1 second and with the tidal volume as the patient's rising chest is visually identified. Ventilation should be provided once every 6 seconds (10 breaths/min) and an advanced airway should be inserted if two or more healthcare providers are avail- able. Hyperventilation should be avoided during rescue breathing. The tidal volume should be maintained at 500 to $600 \mathrm{~mL}$ (6-7 $\mathrm{mL} / \mathrm{kg}$ ) during adult CPR. ${ }^{27-29}$

Mouth-to-mouth ventilation is recommended to provide rescue breathing to the victim. The most common cause of failure in ventilation is inappropriate opening of the airway. If the victim's chest does not rise during the first attempt, ventilation should be conducted again after properly performing the head tilt-chin lift maneuver. When respiration assistance is needed for a victim with spontaneous circulation (e.g., when a strong palpable pulse is detected), ventilation should be performed every 5 to 6 seconds or 10 to 12 per minute. BMV is a method used to provide positive pressure ventilation without an advanced airway. A rescuer provides about 500 to $600 \mathrm{~mL}$ of tidal volume using an adult bag mask. This should be performed for 1 second, and oxygen (concentration greater than $40 \%$, minimum of $10-12 \mathrm{~L} / \mathrm{min}$ ) should be provided if possible. ${ }^{30}$ This procedure is most effective when two or more experienced rescuers use the bag mask.

After the advanced airway is in place, chest compressions should be performed at 100 to 120 per minute without interruption, and ventilation should be performed every 6 seconds (10 breaths/min). Healthcare providers can choose either a bag-mask or an advanced airway while CPR is performed for adult victims of cardiac arrest. A SGA or endotracheal tube can be inserted to serve as an advanced airway.

\section{DURATION OF ON-SCENE CARDIOPULMONARY RESUSCITATION}

Lay rescuers should provide BLS until the arrival of the EMTs. Healthcare providers in the hospital should provide BLS until the arrival of the rapid response team. EMTs should consider hospital transfer if ROSC does not occur after on-site CPR is performed for 6 minutes (three cycles of CPR). If advance resuscitation is possible on the scene, EMTs should consider hospital transfer after performing on-site advanced resuscitation for 10 minutes and may consider extending the time according to the direct medical directions of physicians.

In the case of normal breathing and circulation regardless of unresponsiveness, the victim should be placed in a recovery position to prevent aspiration of foreign body in the mouth.

\section{DEFIBRILLATION}

Despite the importance of an AED, its usage rate is low. ${ }^{31,32}$ The AED is systematically registered and managed to increase its utilization. The emergency medical information website (http://www. 
e-gen.or.kr) and app service provide guidance services and information on AEDs placed in nearby locations. ${ }^{33,34}$ The EMD informs the rescuer of the location of a nearby AED by checking the location of the AED deployed around the site of the cardiac arrest or using a mobile phone app or text message system. ${ }^{35}$

An AED should be used immediately it arrives. If an AED arrives while CPR is being performed, one rescuer should use the AED while the other continues CPR. After taking off the victim's shirt, two pads need to be firmly attached to his/her bare chest. Chest compressions need to be stopped while the AED analyzes the cardiac rhythm of the victim. If defibrillation is required, the AED says, "need defibrillation" and charges the defibrillator by itself. If a voice or screen instruction says, "push the shock button" after charging, the rescuer must ensure that no one touches the patient for safety purposes; the shock button can then be pushed. Chest compressions must be resumed immediately after defibrillation.

Chest compression should not be stopped except while cardiac rhythm is being analyzed or a shock is being delivered. After delivering a shock, chest compression should be restarted immediately. After every 2 minutes of CPR, the cardiac rhythm should be analyzed and the victim's status should be checked. Advanced life support should be started if available.

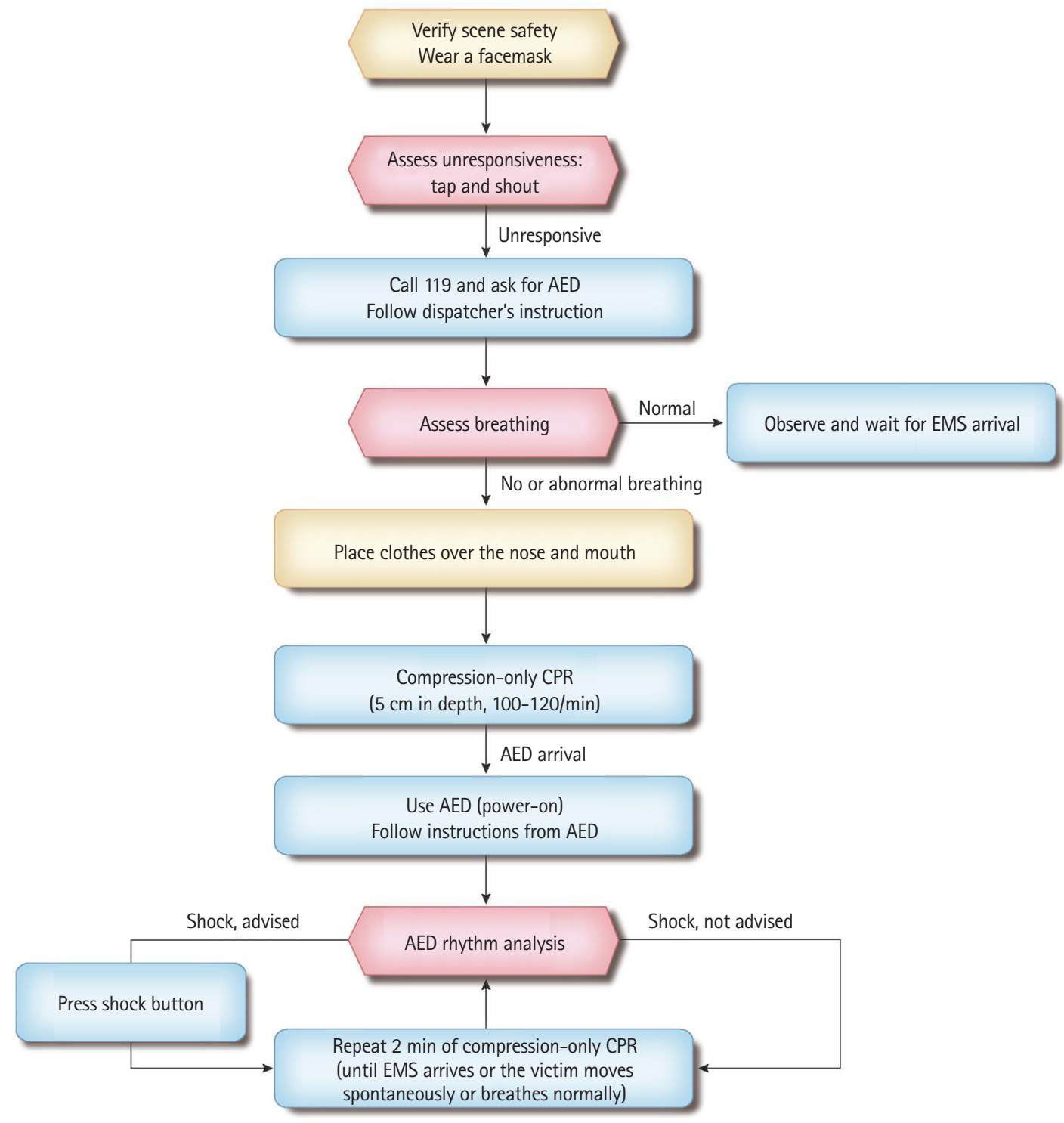

Fig. 4. 2020 Basic life support algorithm for adult out-of-hospital cardiac arrest for lay rescuers during the COVID-19 pandemic. AED, automated external defibrillator; EMS, emergency medical system; CPR, cardiopulmonary resuscitation. 


\section{AIRWAY OBSTRUCTION BY A FOREIGN BODY}

Foreign bodies can cause mild or severe airway obstruction. If an individual with sudden respiratory distress shows signs of airway obstruction, first aid should be administered immediately. If symptoms such as cough, cyanosis, difficulty breathing or speaking, or gripping of the throat are observed, the person should be asked, "Are you choking?". If the victim nods because he/she is not able to speak, it is judged as serious airway obstruction and immediate treatment is required.
Back blows on the back are recommended as a priority treatment for persons with airway obstruction caused by a foreign body or who are unable to cough effectively. It is recommended that the abdominal thrust method be used when back blows are not effective.

If a foreign body is visible in the mouth of an unconscious person, the rescuer should consider removing the foreign body with a finger. If removing by hand is difficult, the rescuer may use instruments such as a laryngoscope and Magill forceps to remove the foreign body.

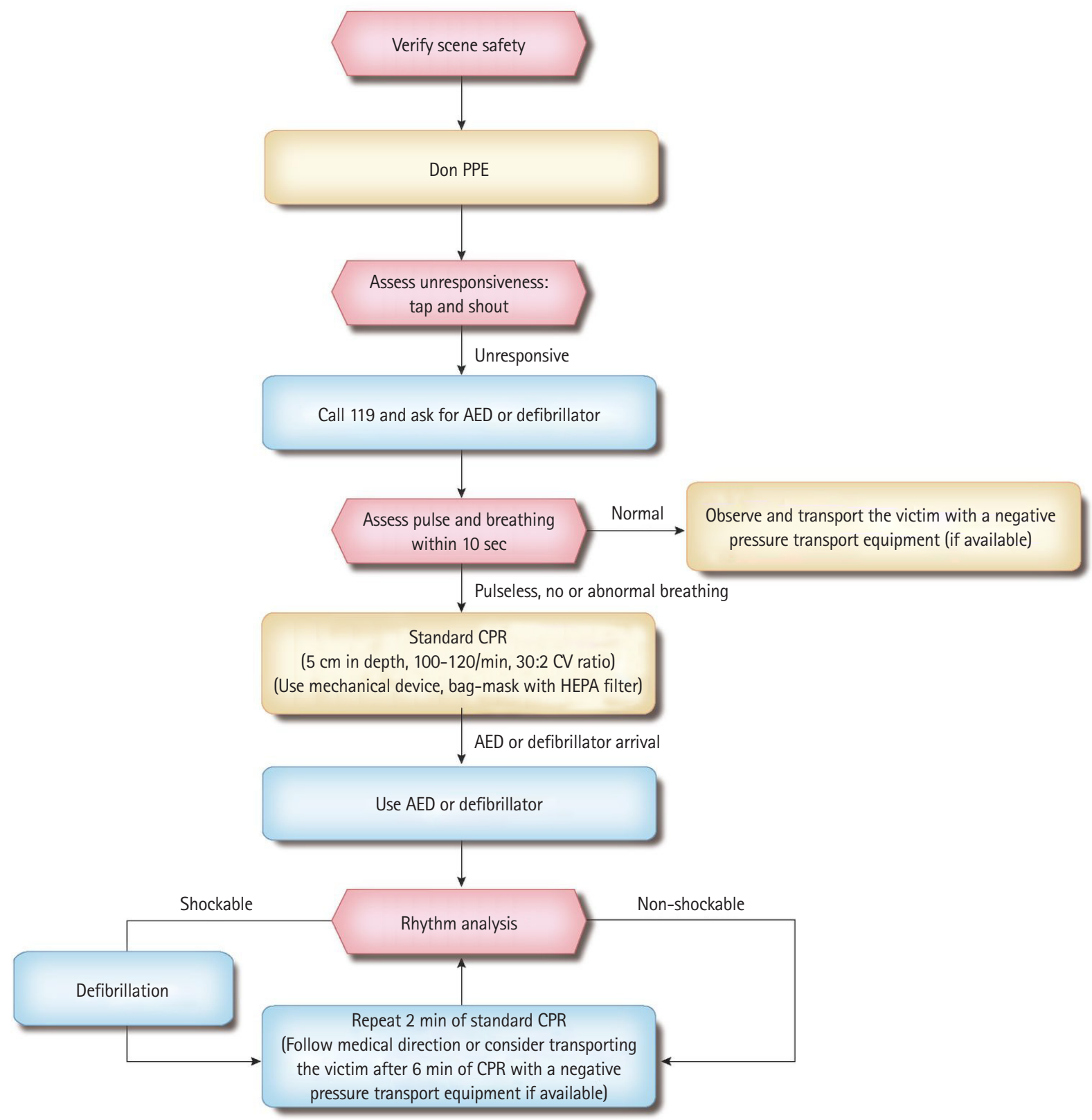

Fig. 5. 2020 Basic life support algorithm for adult out-of-hospital cardiac arrest for healthcare providers during the COVID-19 pandemic. PPE, personal protective equipment; AED, automated external defibrillator; CPR, cardiopulmonary resuscitation; CV, compression-ventilation; HEPA, high-efficiency particulate air. 


\section{DROWNING}

Bystander CPR should be done for victims of cardiac arrest as a result of drowning. If the rescuer is trained and willing to perform, standard CPR, including ventilation, is performed. The use of an AED can be considered for victims of cardiac arrest as a result of drowning. A well-trained aquatic rescue team with appropriate equipment may consider underwater CPR and CPR on a lifeboat. It is suggested that immersion time rather than age, EMS response time, water type (fresh or salt water), water temperature, and witnessed status of the victim, could be used as a prognostic indicator when making search and rescue decisions for drowning patients.

\section{ETHICAL ISSUES RELATED TO CARDIOPULMONARY RESUSCITATION}

The individual's right to decision and self-determination regarding CPR should be respected. However, since CPR must be performed without delay, this may result in unnecessary or unwanted results. ${ }^{36,37}$ Victims of cardiac arrest should be provided CPR immediately; however, there are a few exceptions: 1) when the rescuer is at risk of serious injury; 2) when there are obvious clinical signs of death such as post-mortem stiffness, amputation, or decay; and 3) when there is an indication meaning "Do Not Resuscitate." The rescuer who initiated BLS should continue CPR until one of the following scenarios occur: 1) EMTs arrive and take over; 2) ROSC occurs; 3) the rescuer becomes tired or the situation becomes dangerous for him/her; and 4) a contraindication for CPR is discovered during the procedure.

\section{CONSIDERATION OF THE CORONAVIRUS DISEASE PANDEMIC}

Regarding the current COVID-19 pandemic, chest compressions and CPR can trigger the formation of aerosols, increasing the risk of transmission of infection. Chest compression should be performed with the victim's mouth and nose covered with a face mask or a cloth as there is potential for aerosol generation. If defibrillation is necessary, it should be actively performed while paying attention to the transmission of infection.

Healthcare providers should wear appropriate personal protective equipment, including mask, gloves, gown, and goggles. For ventilation, healthcare providers should use a bag-mask and connect a high-efficiency particulate air filter if possible. When transferring a patient to a hospital, it is recommended that a negative pressure ambulance or a negative pressure transfer device be used if available. Figs. 4 and 5 show the modified CPR algorithm for patients suspected to have COVID-19.

\section{CONFLICT OF INTEREST}

No potential conflict of interest relevant to this article was reported.

\section{ACKNOWLEDGMENTS}

This study was supported by a grant (2020E330300) of the Korean Disease Control and Prevention Agency funded by the Ministry of Health and Welfare, Republic of Korea.

We thank Ms. So Yeong Kim for her assistance with administrative affairs and Mr. Myung Ha Kim for his assistance with literature searches for updating Korean Guidelines for Cardiopulmonary Resuscitation. We also thank the Korean Association of Cardiopulmonary Resuscitation for supporting the process of proofreading.

\section{REFERENCES}

1. Song KJ, Kim JB, Kim J, et al. Part 2. Adult basic life support: 2015 Korean Guidelines for Cardiopulmonary Resuscitation. Clin Exp Emerg Med 2016;3:S10-6.

2. Perkins GD, Walker G, Christensen K, Hulme J, Monsieurs KG. Teaching recognition of agonal breathing improves accuracy of diagnosing cardiac arrest. Resuscitation 2006;70:432-7.

3. Tanaka Y, Taniguchi J, Wato Y, Yoshida Y, Inaba H. The continuous quality improvement project for telephone-assisted instruction of cardiopulmonary resuscitation increased the incidence of bystander CPR and improved the outcomes of outof-hospital cardiac arrests. Resuscitation 2012;83:1235-41.

4. Bang A, Herlitz J, Martinell S. Interaction between emergency medical dispatcher and caller in suspected out-of-hospital cardiac arrest calls with focus on agonal breathing: a review of 100 tape recordings of true cardiac arrest cases. Resuscitation 2003;56:25-34.

5. Becker LB, Pepe PE. Ensuring the effectiveness of communitywide emergency cardiac care. Ann Emerg Med 1993;22:35465.

6. Hupfl M, Selig HF, Nagele P. Chest-compression-only versus standard cardiopulmonary resuscitation: a meta-analysis. Lancet 2010;376:1552-7.

7. Svensson L, Bohm K, Castren M, et al. Compression-only CPR or standard CPR in out-of-hospital cardiac arrest. N Engl J Med 2010;363:434-42. 
8. Iwami T, Kitamura T, Kiyohara K, Kawamura T. Dissemination of chest compression-only cardiopulmonary resuscitation and survival after out-of-hospital cardiac arrest. Circulation 2015; 132:415-22.

9. Lewis M, Stubbs BA, Eisenberg MS. Dispatcher-assisted cardiopulmonary resuscitation: time to identify cardiac arrest and deliver chest compression instructions. Circulation 2013; 128:1522-30.

10. Bohm K, Stalhandske B, Rosenqvist M, Ulfvarson J, Hollenberg J, Svensson L. Tuition of emergency medical dispatchers in the recognition of agonal respiration increases the use of telephone assisted CPR. Resuscitation 2009;80:1025-8.

11. Bobrow BJ, Spaite DW, Vadeboncoeur TF, et al. Implementation of a regional telephone cardiopulmonary resuscitation program and outcomes after out-of-hospital cardiac arrest. JAMA Cardiol 2016;1:294-302.

12. Debaty G, Labarere J, Frascone RJ, et al. Long-term prognostic value of gasping during out-of-hospital cardiac arrest. J Am Coll Cardiol 2017;70:1467-76.

13. Clark JJ, Larsen MP, Culley LL, Graves JR, Eisenberg MS. Incidence of agonal respirations in sudden cardiac arrest. Ann Emerg Med 1992;21:1464-7.

14. Riou M, Ball S, Williams TA, et al. 'She's sort of breathing': what linguistic factors determine call-taker recognition of agonal breathing in emergency calls for cardiac arrest? Resuscitation 2018;122:92-8.

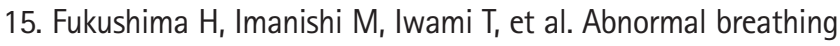
of sudden cardiac arrest victims described by laypersons and its association with emergency medical service dispatcherassisted cardiopulmonary resuscitation instruction. Emerg Med J 2015;32:314-7.

16. Brinkrolf $P$, Metelmann $B$, Scharte $C$, Zarbock $A$, Hahnenkamp $\mathrm{K}$, Bohn A. Bystander-witnessed cardiac arrest is associated with reported agonal breathing and leads to less frequent bystander CPR. Resuscitation 2018;127:114-8.

17. Berg RA, Hemphill $R$, Abella BS, et al. Part 5: adult basic life support: 2010 American Heart Association Guidelines for Cardiopulmonary Resuscitation and Emergency Cardiovascular Care. Circulation 2010;122:S685-705.

18. Stiell IG, Brown SP, Nichol G, et al. What is the optimal chest compression depth during out-of-hospital cardiac arrest resuscitation of adult patients? Circulation 2014;130:1962-70.

19. Stiell IG, Brown SP, Christenson J, et al. What is the role of chest compression depth during out-of-hospital cardiac arrest resuscitation? Crit Care Med 2012;40:1192-8.

20. Zuercher M, Hilwig RW, Ranger-Moore J, et al. Leaning during chest compressions impairs cardiac output and left ven- tricular myocardial blood flow in piglet cardiac arrest. Crit Care Med 2010;38:1141-6.

21. Yannopoulos D, McKnite $S$, Aufderheide TP, et al. Effects of incomplete chest wall decompression during cardiopulmonary resuscitation on coronary and cerebral perfusion pressures in a porcine model of cardiac arrest. Resuscitation 2005;64:36372.

22. Gazmuri RJ, Ayoub IM, Radhakrishnan J, Motl J, Upadhyaya MP. Clinically plausible hyperventilation does not exert adverse hemodynamic effects during CPR but markedly reduces end-tidal $\mathrm{PCO}_{2}$. Resuscitation 2012;83:259-64.

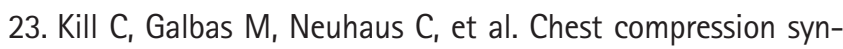
chronized ventilation versus intermitted positive pressure ventilation during cardiopulmonary resuscitation in a pig model. PLoS One 2015;10:e0127759.

24. Abella BS, Alvarado JP, Myklebust $H$, et al. Quality of cardiopulmonary resuscitation during in-hospital cardiac arrest. JAMA 2005;293:305-10.

25. Sayre MR, Berg RA, Cave DM, et al. Hands-only (compressiononly) cardiopulmonary resuscitation: a call to action for bystander response to adults who experience out-of-hospital sudden cardiac arrest: a science advisory for the public from the American Heart Association Emergency Cardiovascular Care Committee. Circulation 2008;117:2162-7.

26. Olasveengen TM, de Caen AR, Mancini ME, et al. 2017 international consensus on cardiopulmonary resuscitation and emergency cardiovascular care science with treatment recommendations summary. Circulation 2017;136:e424-40.

27. Wenzel V, Keller C, Idris AH, Dorges V, Lindner KH, Brimacombe JR. Effects of smaller tidal volumes during basic life support ventilation in patients with respiratory arrest: good ventilation, less risk? Resuscitation 1999;43:25-9.

28. Dorges V, Ocker H, Hagelberg S, Wenzel V, Idris AH, Schmucker P. Smaller tidal volumes with room-air are not sufficient to ensure adequate oxygenation during bag-valve-mask ventilation. Resuscitation 2000;44:37-41.

29. Dorges V, Ocker H, Hagelberg S, Wenzel V, Schmucker P. Optimisation of tidal volumes given with self-inflatable bags without additional oxygen. Resuscitation 2000;43:195-9.

30. Kilgannon $\mathrm{JH}$, Jones $A E_{\text {, Shapiro }} \mathrm{NI}$, et al. Association between arterial hyperoxia following resuscitation from cardiac arrest and in-hospital mortality. JAMA 2010;303:2165-71.

31. Ringh $M$, Hollenberg J, Palsgaard-Moeller $T$, et al. The challenges and possibilities of public access defibrillation. J Intern Med 2018;283:238-56.

32. Myat A, Baumbach A. Public-access defibrillation: a call to shock. Lancet 2019;394:2204-6. 
33. Ringh $M$, Rosenqvist $M$, Hollenberg J, et al. Mobile-phone dispatch of laypersons for CPR in out-of-hospital cardiac arrest. N Engl J Med 2015;372:2316-25.

34. Berglund $E_{1}$ Claesson $A$, Nordberg $P$, et al. A smartphone application for dispatch of lay responders to out-of-hospital cardiac arrests. Resuscitation 2018;126:160-5.

35. Zijlstra JA, Stieglis R, Riedijk F, Smeekes M, van der Worp WE, Koster RW. Local lay rescuers with AEDs, alerted by text messages, contribute to early defibrillation in a Dutch out-of-hos- pital cardiac arrest dispatch system. Resuscitation 2014;85: 1444-9.

36. Guru V, Verbeek PR, Morrison L. Response of paramedics to terminally ill patients with cardiac arrest: an ethical dilemma. CMAJ 1999;161:1251-4.

37. Wiese $\mathrm{CH}$, Bartels UE, Zausig YA, Pfirstinger J, Graf BM, Hanekop GG. Prehospital emergency treatment of palliative care patients with cardiac arrest: a retrolective investigation. Support Care Cancer 2010;18:1287-92. 